\title{
A NEW NUTATION SERIES FOR A RIGID EARTH MODEL
}

\author{
TORSTEN HARTMANN AND MICHAEL SOFFEL ${ }^{1}$ \\ Theoretische Astrophysik, Universität Tübingen \\ Tübingen, Germany
}

\begin{abstract}
A new nutation series for a rigid Earth model was derived from a new and highly accurate tidal potential series. A new second order theory for the nutational amplitudes of rotation-, figure- and angular momentum-axis based on tidal amplitudes is formulated. Amplitudes larger than $0.45 \mu$ as are taken into account leading to a series with 699 terms. The new series may serve as test of other ones that were recently published in the literature.
\end{abstract}

\section{Introduction}

Nutation, together with precession, respresents the motion of some specified Earth axis with respect to some quasi-inertial frame. Up to 1984 , numerical values appearing in nutation series were based upon Woolard's theory (1953) and the relevant Earth axis was the rotation axis. The IAU (1980) nutation series has been adopted as an improvement of Woolard's theory; it is based upon Kinoshita's study (1977) of the nutation of a rigid Earth, which was modified for a deformable Earth using Wahr's theory (1981). Since influences on nutation by elastic and anelastic effects, by oceans and the atmosphere etc. are usually treated as perturbations over those derived for a rigid Earth, a highly precise rigid Earth nutation theory is of tremendous importance especially with respect to the presently achievable accuracy of VLBI measurements. Recently, Zhu and Groten (1989) and especially Kinoshita and Souchay (1990), Souchay and Kinoshita (1996), Roosbeck and Dehant (1995) and Bretagnon (1996) again improved the rigid Earth nutation theory. The goal of our work was first to provide another highly precise rigid Earth nutation theory for the sake of comparisons and

${ }^{1}$ Institut für Planetare Geodaesie, Lohrmann Observatorium, Dresden, Germany

I. M. Wytrzyszczak, J. H. Lieske and R. A. Feldman (eds.), Dynamics and Astrometry of Natural and Artificial Celestial Bodies, 287, 1997.

(C) 1997 Kluwer Academic Publishers. Printed in the Netherlands. 
checks and second to extend and improve the geophysical approach used by Zhu and Groten (1989).

\section{Theory}

Our new nutation series for a rigid Earth model was derived from the new and highly accurate tidal potential series HW95 (Hartmann and Wenzel, 1995a,b). Considered are: the main direct terms from Moon, Sun and planets, indirect effects from planets by modifying the orbits of Moon and Sun, effects from the triaxiality of the Earth and from $J_{3}^{\oplus}, J_{4}^{\oplus}$, geodetic nutation and second order terms.

The HW95 tidal series was derived numerically by a spectral method. Positions of the Earth and tide generating bodies for the period from 1850 to 2150 were taken from DE200. The tidal potential generated by some body $B$ located at $\left(r_{B}, \theta_{B}, \lambda_{B}\right)$ at a point $(r, \theta, \lambda)\left(M_{B}\right.$ is the mass of body $B, \bar{P}$ are the completely normalized Legendre functions)

$$
\begin{aligned}
V_{B}(t)= & G M_{B} \sum_{l=2}^{l_{\max }} \sum_{m=0}^{l} \frac{r^{l}}{r_{B}^{l+1}} \frac{1}{2 l+1} \\
& \times \bar{P}_{l m}(\cos \theta) \bar{P}\left(\cos \theta_{B}\right) \cos \left[m\left(\lambda-\lambda_{B}\right)\right]+\ldots
\end{aligned}
$$

is represented in the form

$$
V_{B}(t)=\sum_{l, m}\left(\frac{r}{a}\right)^{l} \bar{P}_{l m}(\cos \theta) \sum_{i}\left[C_{i}^{l m} \cos \left(\alpha_{i}(t)\right)+S_{i}^{l m} \sin \left(\alpha_{i}(t)\right)\right]
$$

with

$$
C_{i}^{l m}=C 0_{i}^{l m}+t \cdot C 1_{i}^{l m} ; \quad S_{i}^{l m}=S 0_{i}^{l m}+t \cdot S 1_{i}^{l m}
$$

and

$$
\alpha_{i}(t)=m \lambda+\sum_{j=1}^{11} k_{i j} \arg _{j}(t) . \quad\left(k_{i j} \in Z\right)
$$

Here, the fundamental angles $\arg _{j}$ are the usual Doodson variables $\tau=$ mean lunar time, $s=$ mean longitude of the Moon, $h=$ mean longitude of the Sun, $p=$ longitude of lunar parigee, $N^{\prime}=-\Omega, p_{s}=$ longitude of solar perigee and the mean longitudes $L_{i}$ of the planets Mercury, Venus, Mars, Jupiter and Saturn. Note that the relation of $\tau$ to the ususal time $t$ reads

$$
\tau=15^{\circ} / h \cdot t+h-s-\lambda
$$

where $\lambda$ is the longitude of the observing station. The envisaged accuracy of the tidal series is of order $0.1 \mathrm{ngal}=10^{-12} \mathrm{~m} / \mathrm{s}^{2}$, so amplitudes as small 
as $10^{-8} \mathrm{~m} / \mathrm{s}^{2}$ were taken into account. This implies that lunar terms up to degree 6 and solar terms up to degree 3 were considered. The HW95 tidal series comprises 11452 luni-solar and 1483 direct planetary terms. Presently, it represents the most precise tidal series available.

It is well known that in the system of principal axes with principal moments of inertia $(A, B, C)$ the $l=2$ part of the torque acting on the rigid Earth can be written as

$$
\mathrm{T}=\sum_{i}\left(\begin{array}{c}
(C-B)\left[S_{i}^{21} \cos \alpha_{i}-C_{i}^{21} \sin \alpha_{i}\right] \\
(A-C)\left[C_{i}^{21} \cos \alpha_{i}+S_{i}^{21} \sin \alpha_{i}\right] \\
(B-A)\left[S_{i}^{22} \cos \alpha_{i}+C_{i}^{22} \sin \alpha_{i}\right]
\end{array}\right)
$$

and similar relations hold for the torque for $l=3,4, \ldots$. Then, the dynamical and kinematical Euler equations for the figure axis are solved by iteration up to the desired accuracy (details will be published elsewhere; see also Hartmann, 1996). The motions of angular momentum axis and rotation axis are then obtained by corresponding Oppolzer terms. The Euler angles $(\theta, \psi, \varphi)$ for the figure axis are then written as

$$
\begin{aligned}
\theta & =\omega_{A}+\Delta \epsilon_{F} \\
\psi & =\psi_{A}+\Delta \psi_{F} \\
\varphi & =\chi_{A}+\Delta \chi_{F}+\operatorname{GAST}(t)
\end{aligned}
$$

where $\omega_{A}$ is the precession in obliquity, $\psi_{A}$ the precession in longitude and $\chi_{A}$ the planetary precession. $\Delta \psi_{F}$ and $\Delta \epsilon_{F}$ are the nutation angles with respect to some mean fixed ecliptic. For the sake of comparisons they are transformed to nutation angles with respect to the mean ecliptic of date $\Delta \psi_{D}, \Delta \epsilon_{D}$.

The truncation threshold for the nutation amplitudes was chosen to be $0.45 \mu \mathrm{as}$, which is smaller by one order of magnitude than that from the KS90 nutation model. To be more precise, the condition for keeping a term was

$$
\left|\sin \varepsilon_{0} \cdot \Delta \psi\right| \geq 0.45 \mu \text { as } \text { or }|\Delta \varepsilon| \geq 0.45 \mu \text { as. }
$$

The total error is larger by at least a factor of 20 . To keep that error under the truncation threshold of $0.45 \mu$ as for including the terms all nutation amplitudes are given to a resolution of $0.01 \mu$ as.

\section{Results}

Since the solutions to first order contain the resonance term $\Delta \omega_{i}=\omega_{i}-\Omega_{\oplus}$ in the denominator tides with $\Delta \omega_{i} \sim 0$ (essentially the $K_{1}$-tide) determine the precession. Using the tidal model HW95 (and the separately derived 
TABLE 1. Planetary precession (in $\mu \mathrm{as} / \mathrm{yr}$ ), $H_{\mathrm{dyn}}=3.273792489 \cdot 10^{-3}$.

\begin{tabular}{lrrrrrr}
\hline body & $\Delta \dot{\psi}_{H}$ & $\Delta \dot{\psi}_{W}$ & diff. & $\Delta \dot{\varepsilon}_{H}$ & $\Delta \dot{\varepsilon}_{W}$ & diff. \\
\hline Mercury & 3.700 & 3.697 & 0.003 & -0.089 & -0.088 & -0.001 \\
Venus & 181.586 & 181.565 & 0.021 & -16.799 & -16.813 & 0.014 \\
Mars & 6.001 & 5.998 & 0.003 & 0.356 & 0.356 & 0.000 \\
Jupiter & 117.050 & 117.068 & -0.018 & 2.810 & 2.804 & 0.006 \\
Saturn & 5.211 & 5.188 & 0.023 & 0.220 & 0.219 & 0.001 \\
Uranus & 0.098 & 0.100 & -0.002 & 0.000 & 0.001 & -0.001 \\
Neptune & 0.028 & 0.029 & -0.001 & 0.000 & 0.001 & -0.001 \\
\hline Total & 313.674 & 313.645 & 0.029 & -13.502 & -13.520 & 0.018 \\
\hline
\end{tabular}

values for the $K_{1}$-tide due to Uranus and Neptune) values for the planetary precession were derived. Results are presented in Table 1; they show excellent agreement with the numbers given by Williams (1995).

Results for the luni-solar precession in longitude are in good agreement with recent results from other authors. For the precession in obliquity this, however, is not the case. The accuracy of our method in this case is relatively poor due to the existence of a smaller tide lying very close to the $K_{1}$-tide.

\subsection{THE NUTATION TERMS AT FIRST ORDER}

\subsubsection{Direct planetary nutation terms}

The direct planetary nutation terms have been computed e.g. by Vondrak (1983), Hartmann and Soffel (1994), and Williams (1995). It is very instructive to use these results for some statements about comparisons in general. Hartmann and Soffel (1994) computed the nutation due to the direct planetary effect using a simplified version of the theory outlined here. The planetary tidal potential used at that time was based on the numerical ephemerides DE102 but differences to DE200 are very small. The threshold was $2.5 \mu$ as and the same for $\Delta \psi$ and $\Delta \varepsilon$ without the factor of $\sin \varepsilon_{0}$. A term by term comparison with values from Williams (1995) gives for the maximum difference, the sum of the absolute differences and the root mean squares (rms) error the values indicated in Table 2. Computing a time series over 90 years starting from J2000 and evaluating the differences in time domain leads to the differences displayed in Table 3. It is important to note that the rms values are about one order of magnitude larger than those from the comparison of individual terms. Furthermore, the maximum and minimum differences nearly reach the sum of the absolute differences of the 
TABLE 2. Term by term comparison: direct planetary nutation H96NUT - WILLIAMS (1995), values in $\mu$ as.

\begin{tabular}{lrrrr}
\hline & $\Delta \psi_{\text {sin }}$ & $\Delta \psi_{\text {cos }}$ & $\Delta \varepsilon_{\text {sin }}$ & $\Delta \varepsilon_{\text {cos }}$ \\
\hline Max. & 2.31 & 3.77 & 1.26 & 1.50 \\
$\sum||$ & 13.21 & 16.14 & 6.12 & 5.87 \\
rms & 0.35 & 0.53 & 0.18 & 0.20 \\
\hline
\end{tabular}

TABLE 3. Comparison H96NUT WILLIAMS (1995) in time domain: direct planetary nutation, values in $\mu$ as.

\begin{tabular}{rrrrr}
\hline & Max & Min & Mean & Rms \\
\hline$\Delta \psi$ & 8.26 & -12.14 & -1.47 & 3.53 \\
$\Delta \varepsilon$ & 4.75 & -1.34 & 1.57 & 1.82 \\
\hline
\end{tabular}

individual terms. As already mentioned in Hartmann and Soffel (1994) the direct planetary nutation can be computed from a tidal potential with very high accuracy.

\subsubsection{Nutation term due to $J_{3}$ and $J_{4}$}

The nutation terms due to the higher order parts of the tidal potential have already been given in Hartmann, Williams and Soffel (1996). As an extension the largest solar term due to $J_{3}^{\oplus}$ has argument $F-D+\Omega$, a period of 365.229 days and amplitudes of $-0.26 \mu$ as and $-0.22 \mu$ as for $\Delta \psi$ and $\Delta \varepsilon$, respectively. Hence, this term is under our threshold of $0.45 \mu$ as.

\subsubsection{Nutation terms due to the triaxiality of the Earth}

The so-called triaxiality terms result from the difference in the equatorial moments of inertia $A$ and $B$ (or equivalently from the geopotential coefficients $C_{22}$ and $S_{22}$; not to be confused with the sectorial tidal amplitudes). Since that difference is small and the corresponding formulas show no resonance denominator only a few terms play a role (period: half a day). They are given in Table 4 where $2 \tau$ has to be added to the argument.

It should be mentioned that an updated value (from GEM-T3) for the factor $R$ defined in KS90 has been used here (KS90: $3.272 \cdot 10^{-3}$, H96NUT: $\left.3.353598 \cdot 10^{-3}\right)$. A comparison with KS90 shows that some of their terms 
TABLE 4 . The triaxiality terms, values in $\mu$ as.

\begin{tabular}{rrrrrrrrrrl}
\hline$l_{m}$ & $l_{s}$ & $F$ & $D$ & $\Omega$ & period $(\mathrm{d})$ & $\Delta \psi$ & $\Delta \psi_{\mathrm{KS} 90}$ & $\Delta \varepsilon$ & $\Delta \varepsilon_{\mathrm{KS} 90}$ & body \\
\hline 0 & 0 & -3 & 0 & -3 & 0.528 & 0.00 & -5 & 0.00 & 0 & $\mathrm{M}$ \\
-1 & 0 & -2 & 0 & -2 & 0.527 & -5.94 & 0 & 2.36 & 0 & $\mathrm{M}$ \\
0 & 0 & -2 & 0 & -1 & 0.518 & -5.52 & -5 & 2.20 & 0 & $\mathrm{M}$ \\
0 & 0 & -2 & 0 & -2 & 0.518 & -29.25 & -26 & 11.63 & 10 & $\mathrm{M}$ \\
-1 & 0 & 0 & 0 & 0 & 0.508 & 2.17 & 0 & -0.86 & 0 & $\mathrm{M}$ \\
0 & 0 & -2 & 2 & -2 & 0.500 & -12.23 & -12 & 4.87 & 5 & $\mathrm{~S}$ \\
0 & 0 & 0 & 0 & 0 & 0.499 & 25.10 & & -9.98 & & $\mathrm{M}$ \\
0 & 0 & 0 & 0 & 0 & 0.499 & 11.62 & 36 & -4.62 & -14 & $\mathrm{~S}$ \\
0 & 0 & 0 & 0 & -1 & 0.499 & 4.98 & -5 & -1.98 & 0 & $\mathrm{M}$ \\
0 & 0 & 2 & -2 & 2 & 0.497 & 0.00 & 5 & 0.00 & 0 & $\mathrm{M}$ \\
1 & 0 & 0 & 0 & 0 & 0.490 & 1.95 & 0 & -0.77 & 0 & $\mathrm{M}$ \\
\hline
\end{tabular}

are not correct. In Table $4 \mathrm{M}$ (S) indicates that the corresponding term results from the action of the Moon (Sun).

\subsubsection{Luni-solar nutation terms at first order due to $J_{2}$}

The direct luni-solar nutation terms, i.e.those which can be represented solely by the Delaunay arguments $l_{m}, l_{s}, F, D$ and $\Omega$, and the indirect planetary nutation terms which require the mean longitude of at least one planet are treated in the same way (in contrast to KS90 which use different ephemerides therefore requiring $p_{A}$ as additional argument for the indirect planetary nutation terms).

\subsubsection{The main luni-solar terms}

Comparing the results for the main luni-solar terms with those of KS90 one finds an overall good agreement. However, for about 30 terms the difference in longitude exceeds $10 \mu$ as and the same holds for the difference in obliquity. These differences result from difficulties related with our geophysical approach: i) very closely lying tides might not be resolved easily on a purely numerical basis and ii) because of resonance denominators very weak tides might contribute at a certain level of accuracy if they lie sufficiently close to the $K_{1}$ tide. These problems might, however, be solved with additional investigations.

\subsection{THE NUTATION TERMS AT SECOND ORDER}

Our theory also describes second order terms which include the geodetic nutation (e.g. Fukushima, 1991) among others (like the precession on nutation etc.). It must be stressed that these terms cannot be compared directly 
with second order terms of other nutation series since they differ by definition. To demonstrate this fact let us consider the so-called $J_{2}$-tilt effect. The $J_{2}$ coefficient of the Earth slightly tilts the orbit of the Moon with respect to the ecliptic. This is - due to the inclusion of the corresponding force and the numerical integration of the equations of motion of all bodies in the solar system in the numerical ephemerides DE200 - implicitly included in the tidal potential HW95 and therefore also in the nutation model H96NUT. Other theories which use analytical ephemerides without this force have to include the $J_{2}$-tilt effect separately. However, the total nutation amplitudes should be directly comparable.

Acknowledgements. This work was supported by the Deutsche Forschungsgemeinschaft (DFG).

\section{References}

Bretagnon, P.: 1996, this volume.

Fukushima, T.: 1991, Astron. Astrophys. 244, L11-L12.

Hartmann, T. and Soffel, M.: 1994, Astron. J. 108, 1115-1120.

Hartmann, T. and Wenzel, H.-G.: 1995a, Bulletin d'Information des Marées Terrestres 123, 9278-9301.

Hartmann, T. and Wenzel, H.-G.: 1995b, Geophys. Res. Lett. 22, 3553-3556.

Hartmann, T., Williams, J. and Soffel, M.: 1996, Astron. J. 111, 1400-1404.

Hartmann, T.: 1996, "Hochgenaue Nutationsbewegung einer starren Erde aus einer verbesserten Gezeitenpotentialentwicklung", Ph.D. thesis, Univ. of Tübingen, Germany (in German).

Kinoshita, H.: 1977, Celest. Mech. 15, 277-326.

Kinoshita, H. and Souchay, J.: 1990, Celest. Mech. 48, 187-265 (called KS90 in the text). Roosbeek, F. and Dehant, V.: 1995, "A harmonic development of the rigid Earth nutations using an analytical method", in preparation.

Souchay, J. and Kinoshita, H.: 1995, Astron. Astrophys., in press.

Vondrak, J.: 1983, Astron. Inst. Czechosl. 34, 184-190 and 311-316.

Williams, J.G.: 1994, Astron. J. 108, 711-724.

Williams, J.G.: 1995, Astron. J. 110, 1420-1426.

Woolard, E.W.: 1953, Astron. Papers Am. Ephem. 15, Part 1.

Zhu, S.Y. and Groten, E.: 1989, Astron. J. 98(3), 1104-1111. 